\title{
SEMI-ISOMORPHISMS OF CERTAIN INFINITE PERMUTATION GROUPS
}

W. R. SCOTT

Let $X$ and $Y$ be infinite cardinal numbers, $S(X)$ the full symmetric group on a set of cardinal $X, A(X)$ the alternating group of finite even permutations on the same set, and $S(X, Y)$ the subgroup of $S(X)$ of all permutations moving fewer than $Y$ elements.

A semi-automorphism of a group $G$ is a permutation $T$ of $G$ such that $(x y x) T=(x T)(y T)(x T)$ for all $x, y \in G$. Semi-isomorphism is defined similarly. Dinkines [1] and Herstein and Ruchte [2] showed that any semi-automorphism of $S(X, Y)$ or $A(X)$ was either the restriction $T$ of an inner automorphism of $S(X)$, or was of the form $T(-I)$ where $x(-I)=x^{-1}$ for all $x$. Theorem 11.4 .6 of [3] states that every automorphism of any group $G$ such that $A(X) \subset G \subset S(X)$ is the restriction of an inner automorphism of $S(X)$. In the present paper, we prove the common generalization of these two theorems whose statement is obvious. (See the corollary at the end.)

LEMma. If $Q$ is a subset of $A(X)$ containing all 3-cycles and such that $x, y \in Q$ imply $x y x \in Q$, then $Q=A(X)$.

Proof. ${ }^{1}$ If $x \in A(X)$, then $x=c_{1} c_{2} \cdots c_{n}$, where the $c_{i}$ are 3 -cycles. If $n=1$, then $x \in Q$. Induct on $n$. Since

$$
x=c_{1} \cdots c_{n}=c_{1}^{-1}\left(c_{1}^{-1}\left(c_{2} \cdots c_{n}\right) c_{1}\right) c_{1}^{-1}
$$

and the middle factor is the product of $n-13$-cycles $c_{1}^{-1} c_{i} c_{1}$, it follows by induction that $x \in Q$.

Theorem. Let $X$ be an infinite cardinal number, $G$ and $H$ subgroups of $S(X)$ containing $A(X)$, and $T$ a semi-isomorphism of $G$ onto $H$. Then either

(1) $T$ is induced by conjugation by an element of $S(X)$, or

(2) $T$ is the product of a mapping of type (1) mapping $G$ onto $H$, and the mapping $-I$ of $H$ onto $H$.

Proof. Since the center of $H$ is 1 , it follows that $T$ preserves order and powers [1, Lemma 1]. Let $S\left(S^{\prime}\right)$ be the subgroup generated by all elements of order 2 in $G(H)$. Since $A(X)$ is simple, $S$ and $S^{\prime}$ each contain $A(X)$. Call elements $x, y \in G, S$-conjugate iff $x=y^{8}$ for some

Received by the editors August 16, 1968.

1 This brief proof is due to Fletcher Gross. 
$s \in S$. Define $S^{\prime}$-conjugacy similarly. Then $S$ - and $S^{\prime}$-conjugacy are equivalence relations. Moreover, if $x$ is $S$-conjugate to $y$, then $x$ $=u_{n} \cdots u_{1} y u_{1} \cdots u_{n}$ with $o\left(u_{i}\right)=2$, so that

$$
x T=\left(u_{n} T\right) \cdots\left(u_{1} T\right)(y T)\left(u_{1} T\right) \cdots\left(u_{n} T\right),
$$

$o\left(u_{i} T\right)=2$, and $x T$ and $y T$ are $S^{\prime}$-conjugate. Since the inverse of a semi-isomorphism is also a semi-isomorphism, the converse is also true. Therefore $T$ carries an $S$-conjugate class onto an $S^{\prime}$-conjugate class.

Let $M$ be the set of 3-cycles. Then

(3) $M$ is an $S$-conjugate class of $G$.

(4) All elements of $M$ have order 3.

(5) $\operatorname{Max} o(x y x)=5$ for $x, y \in M$.

By earlier remarks, $M T$ satisfies these conditions with $H$ instead of $G$ and $S^{\prime}$ instead of $S$. If $x=(123)(456) \in M T$, then conjugation by an appropriate $s \in A(X) \subset S^{\prime}$ gives $y=(132)(478) \in M T$. Then

$$
x y x=(123)(46785)
$$

contrary to (5). If $x=(123)(456)(789) \cdots \in M T$, then conjugation by some $s \in S^{\prime}$ yields $y=(132)(457)(689) \cdots \in M T$. But then

$$
x y x=(123)(48)(59) \cdots,
$$

contrary to (5). By (4), it follows that all elements of $M T$ are 3cycles. Hence, by (3), $M T=M$.

Let $Q=\{x \in A(X) \mid x T \in A(X)\}$. If $x, y \in Q$, then $x y x \in Q$. By the lemma, $Q=A(X)$, that is $A(X) T \subset A(X)$. Using $T^{-1}$ instead of $T$, we have $A(X) T=A(X)$. Thus $T \mid A(X)$ is a semi-automorphism. By [1] or [2], $T \mid A(X)$ is either an automorphism or an anti-automorphism. But all automorphisms of $A(X)$ are of the form $T_{z}$ where $T_{z}$ is conjugation by some element $z \in S(X)$ (see, for example, [3, Theorem 11.4.8]). If $T$ is an anti-automorphism on $A(X)$, then $T(-I)$ is an automorphism, hence $T(-I)=T_{z}$ and $T=T_{z}(-I)$ on $A(X)$.

Let $U=T T_{z}^{-1}$ or $T(-I) T_{z}^{-1}$ in the above two cases. Then $U$ is a semi-isomorphism of $G$ which is the identity on $A(X)$. The theorem will follow if we can show that $U$ is the identity on $G$.

Suppose that there is an $x \in G$ such that $x U \neq x$. We assert that $x$ can be chosen so that it fixes at least 5 letters. If this is false, then choose $x$ so that it fixes the maximum possible number of letters (at most 4). If $x$ contains an $n$-cycle $n \geqq 3$, we can assume that $x$ $=(\cdots 123 \cdots) \cdots$, in which case $(123) x(123)$ fixes all letters fixed by $x$ and the letter 3 in addition. In the other case, $x$ is a product of disjoint 2-cycles, say $x=(12)(34) \cdots$; then $(123) x(123)$ again 
fixes 3 and all letters fixed by $x$. Since for $y=(123),(y x y) U=y(x U) y$ $\neq y x y$, we have a contradiction in either case. Hence, as asserted, $x$ can be chosen so that it fixes at least 5 letters.

Now let $x$ be any element of $G$ fixing at least 5 letters. We assert that $x U$ fixes the same letters as $x$. Suppose, in fact, that $x$ fixes 1,2 , 3,4 , and 5 , but that $x U$ moves 1 . Then, changing notation if necessary, $1(x U) \neq 1,2,3$, or 4 . Now

$$
\begin{gathered}
x U=[(12)(34) x(12)(34)] U=(12)(34)(x U)(12)(34), \\
(x U)(12)(34)=(12)(34)(x U) .
\end{gathered}
$$

But the left side sends 1 into $1(x U)$, while the right sends it into $2(x U)$. This contradiction proves that $x U$ fixes all letters fixed by $x$. Consideration of $U^{-1}$ shows that $x U$ fixes the same letters as $x$.

Let $x$ fix at least 5 letters, and $x U \neq x$. For some letter $i, i x=j$, $i(x U)=k, k \neq j$. The preceding paragraph implies that $i \neq j$. Let $r \neq i$ or $j$. Now the element $(r i j) x$ (rij) fixes $r$ and all letters fixed by $x$, hence at least 5 letters altogether. However $(r i j)(x U)(r i j)=[(r i j) x(r i j)] U$ does not fix $r$. This contradicts the preceding paragraph. Thus the theorem is true.

Corollary. If $A(X) \subset G \subset S(X)$ and $T$ is a semi-automorphism of $G$, then there is an element $z \in N_{S(X)}(G)$ such that either $T$ is the automorphism $T_{z}$ (induced by conjugation by $z$ ) or the anti-automorphism $T_{z}(-I)$.

\section{BIBLIOGRAPHY}

1. Flora Dinkines, Semi-automorphisms of symmetric and alternating groups, Proc. Amer. Math. Soc. 2 (1951), 478-486.

2. I. N. Herstein and M. F. Ruchte, Semi-automorphisms of groups, Proc. Amer. Math. Soc. 9 (1958), 145-150.

3. W. R. Scott, Group theory, Prentice-Hall, Englewood Cliffs, N. J., 1964.

UNIVERSITY OF UTAH 